\title{
Software of Information-Measurement System for Standardless Diagnostic of Composite Materials
}

\author{
V. S. Eremenko, A. V. Pe reidenko* \\ National Aviation University, Information-Measuring Systems Department, Kiev, 03680, Ukraine
}

\begin{abstract}
This article is devoted to software realization via NI Lab VIEW 2011 for system of the standardless diagnostic of technical objects. The solution requires methods that are fast and effic ient in diagnostics, adapted for usage condition changes, oriented for wide set of objects under control and without changes in the main software structure. The structure and main modules of developed software is represented in the article. Developed software advantages are in its architecture flexibility, high performance and reliability of data signal processing, human-engineered interface. The software of standardless diagnostics system is based on neural network classifier which provides flexible and stable knowledge base about possible classes of defects, performs effective operations with high dimensional data vectors, adapts its architecture for solving new tasks and provides the high reliability of control. The classifier based on hybrid neural network, ART-2 and Fuzzy-A RT neural networks for classification of defects in honeycomb panels were introduced and investigated. Described classifier during the training can automatically change its settings, reaching the highest reliability of the control, detect and classify subsurface defects in honeycomb panels with high reliability and accuracy, as well as defects that are located on the back side of the cladding with plottage larger than $2 \mathrm{~cm} 2$ and thickness of composite panel equal to $12.8 \mathrm{~mm}$. The reliability of the nondestructive testing via specified classifier is more than $95 \%$. Results of the developed special software practical usage for honeycomb panels' technical state classification were represented
\end{abstract}

Keywords Standardless Diagnostic, System of Nondestructive Testing, Neural Net work, NI Lab VIEW 2011, Honeycomb Panel

\section{Introduction}

There are many methods and systems to diagnosis and classify the technical state of objects under control (OUC) [1-4]. However, their usage does not allow perform high quality of standardless diagnostics of products that have complicated structure: composite material products, blades of gas turbine engines, bandage of electric locomotive wheelset, etc. Standardless diagnostics of such objects should be performed with methods which would allow accumulate retrieved in formation about new defects, expand existed base of defects and increase diagnostics system precis ion without restarting (but in runtime). The other problem is the high number of informative parameters that describe the technical state of object under control. In such cases the number of dimensions of the features space (that used for decision rules making) is high. It is difficult to use statistical methods for these purposes, because the analysis of high dimensional probability distribution functions should

* Corresponding author:

apereedenko@gmail.com (A.V. Pereidenko)

Published online at http://journal.sapub.org/se

Copyright (C) 2012 Scientific \& Academic Publishing. All Rights Reserved be performed. It results in significant usage of hardware and time resources.

Nowadays the developing of standardless diagnostics methods for controlling the technical state of products with complicated structure is actual problem. There are many researches devoted to problems of information-measuring systems construction and such issues as improvement of efficiency, accuracy, noise immunity, reliability, performan ce, and software poly morphism of these systems [5-8]. The solution requires methods that are fast and efficient in OUC diagnostics, adapted for usage condition changes, oriented for wide set of OUC and without changes in the main software structure[5].

Software of such diagnostics system must include next two blocks:

- Diagnostics block. Its function is to find the parts with defects (anomalies) and/or to form studying samples for classification problems. Tasks of this block could be divided on three groups:

a. indicating diagnostics characteristics for different OUCs states (valid or invalid);

b. forming learning samples for every object state;

c. decision rule making based on input vector of diagnostics characteristics analysis. 
- Classification block. There are three groups of its functions:

a. indicating diagnostics characteristics for different OUCs states (damage levels, defect types, etc.);

b. forming features space and separating hyperplanes;

c. classifying input informative signals using different classification methods.

Among the most popular methods of standardless diagno stics and classification (based on separating hyperplanes [9-12], statistical analysis of the spectral densities [13-16] or analysis via artificial neural networks[17-21]) there are methods based on artificial neural networks (NN). Usage of such methods has next advantages: high noise immunity, high diagnostics efficient[19-23], etc. It allows increase the control reliability and performance, reduce systemrealizatio $\mathrm{n}$ cost and hardware resources. Neural networks give opportunity to perform clustering analysis[24,25], build comp licated separating hyperplanes and perform classificati on of objects under control[26]. They learn new information and expand existing knowledge base about nomenclature of possible classes without information loss.

There is a lot of publications[19-23] in which the questions of efficient $\mathrm{NN}$ applying for tasks of objects under control state and defects determining, signal registration and classification, cluster analysis, etc. are discussed. For selection an optimal structure of NN that will be used as a part of informational-measuring system for nondestructive testing, it was investigated a set of such popular neural network architectures as Kohonen NN[25], multilayer perceptron[26], special hybrid $\mathrm{NN}[9,10]$, NN of adaptive resonance theory (ART nets), ART-2 net $[17,27,28]$ and Fuzzy-ART[18,29]. As the result of analysis of these architectures the decision to use as the classifier core the ART-2, Fuzzy-ART and the hybrid neural network (which based on Kohonen NN and mu ltilayer perceptron) was made. All these neural networks allow retrieve the highest control reliability during diagnostics of products from composite materials $[17,18]$.

\section{Problem Solving}

For solving described problems authors have developed special information-measurement system (software and some modules of hardware) for standardless diagnostics of composite materials. The software of standardless diagnosti cs system is based on neural network classifier which provides flexible and stable knowledge base about possible classes of defects, performs effective operations with high dimensional data vectors, adapts its architecture for solving new tasks and provides the high reliability of control. Neural networks also provide the invariance of system usage, efficient input data processing, analysis and classification without dependence on diagnostics and data collecting methods.

There are different software packages such as MathLab [30], NeuroSolution[31], NeuroPro[32], STATISTICA[33] and others that provide the ability to work with mathematical apparatus of neural networks. These packages could be used for solving the wide verity of problems. But in other hand they restrict the available $\mathrm{NN}$ architecture usage and do not allow performing adjustment on neuron level, creating add-ons and developing new learning and functional algorith ms of NN. So me arch itectures of neural networks are absent in such software packages. Based on this fact software development of the nondestructive testing system was performed by authors in NI Lab VIEW 2011 development environment. This environment provides graphical programming language $\mathrm{G}$ and does not restrict the $\mathrm{NN}$ model implementation. NI Lab VIEW [34,35] allows developing new learning and functional $\mathrm{NN}$ algorithms, creating new types of $\mathrm{NN}$ or merging/joining existing nets in one functional system. Also it is possible to use existing programming modules and DLLs. LabVIEW 2011 environment performs parallel execution of algorithms which increases the performance of created nondestructive testing system software.

The nondestructive testing system software is developed by authors like a set of independent modules because of the need to solve the problem of standardless diagnostics for different object under control types with usage of only one system with possible minor changes for each specific task (difference could be in changing few existing functions or including new one for specific diagnostics tasks). It allows connecting and integrating created earlier libraries and modules (which were developed with high level languages) in the system core without its significant changes. This approach permits adding new or excluding unnecessary functionality, performing fast reconfiguration, modernizatio $\mathrm{n}$, adaptation, etc. without any complexity.

Developed system for OUC technical state diagnostics is oriented on work with standard devices for retrieving initial information (defectoscopes) as well as with specialized data retrieving devices (for specific tasks and OUC) which based on analog-digital converters (ADC).

This is achieved by using generalized input-output module (I/O Module) which is developed by authors and interacts with defectoscopes or with specialized data retrieving systems. In the developed standardless diagnostics system structure (Fig. 2) there are two main parts: hardware (includes a set of sensors, data acquisition unit and $\mathrm{I} / \mathrm{O}$ Module) and software.

\section{Hardware Part}

The system's hardware part consists next blocks:

- Set of sensors - initial physical para meter transformers into electric signal;

- Data acquisition unit - data acquisition platform cDAQ or cRIO (National Instruments) with set of ADC and specialized modules or specialized defectoscope that is used for experimental data acquisition; 
- I/O Module - interacts with defectoscopes or with specialized data retrieving systems and transmits data for subsequent data processing.

In case of use cDAQ platform (Fig. 1) communication with PC is performed via USB 2.0 interface. Channel capacity is equal to $3.2 \mathrm{Mb} / \mathrm{s}$. A set of ADC modules varies depending on specific tasks (required number of channels, sampling rate, dynamic range of input signal, etc). In the developed system the ADC NI 9215 is used. It has 4 simultaneously sampled analog inputs, $100 \mathrm{kS} / \mathrm{s}, \pm 10 \mathrm{~V}$ dynamic range, 16-bit resolution and 40 to $70{ }^{\circ} \mathrm{C}$ operating range.

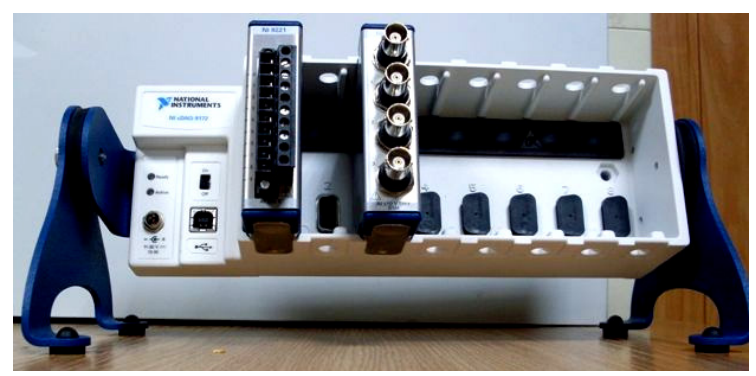

Figure 1. cDAQ data acquisition platform

Like a physical method for detecting initial information about object under control (detect defects of composite materials) the method of low-velocity impact[36] has been used. The method of low-velocity impact is based on measuring parameters of impact on the object under control. At the moment when firing-pin with a kinetic energy $A$ affects on the object under control an impulse of shock interaction force arises. This impulse is characterized by an amp litude, duration, waveshape and brings rich information about technical state of object under control. The main part of information about technical state of OUC is concentrated in signal waveshape. The method of low-velocity impact is effective for diagnostics of fatigue damage, bundle defects, link breaks, defects which begin to born and also crack-like defects with millimeter size etc.

\section{Software Part}

Interaction between $\mathrm{I} / \mathrm{O}$ Module and $\mathrm{PC}$, information stream organization, data processing algorithms realization and displaying the result was implemented by authors in software part of the system (mathematical support, dataware and $\mathrm{I} / \mathrm{O}$ Module software). The software architecture is displayed on Fig.3.

There are next main modules of system software:

- ADC control module. This module includes units for configuring ADC parameters (sampling rate, dynamic range of input signals, number of ADC channels, synchronization source, etc.).

- System's regime control module. It is used for configuring such parameters of the system regime like the input data source (defectoscope, ADC or physical/optical data storage), system regime, etc.

- Data storages interaction control module. It allows reading/writing data fro $\mathrm{m} /$ to data storage. It also forms array or subarray of necessary informative parameters for importing them to other diagnostics systems or for creating reports. In case when reading operation is performed this module forms the array fro $m$ the stored data and represents it in required format.

- Reporting facilities and system output control modules. These modules perform data transformation to convenient user-friendly format in case of data displaying or to required data storage format in case of data saving. Also it is possible to display or save on storage parameters of neural networks which are used in the system.

- Control module for system database. It allows $\mathrm{read} /$ write set of formed classes during system work as well as their additional specific parameters.

- Control module for neural networks. It allows user to choose the type of neural network that will be used for data processing and classification and setup its parameters. This module contains units which perform class forming of OUC, finding new classes, anomalies, and converting the neural network output to user-friendly format.

- Data buffer control module. This module writes retrieved data from ADC or defectoscope to array which further could be processed or saved for processing in future by current or another diagnostics system.

- Data processing module. It allows choosing the necessary diagnostics characteristics which the most informative for current case and use them instead the full set of characteristics. In some cases the data vectors of small dimension size could be used. For efficient diagnostics system work the input data should be preprocessed to in specific way. That's why in this module the necessary algorith ms are executed.

Fig. 4 illustrates the interface of developed system. It contains control elements for ADC setting up, preparing and normalizing input data, choosing neural network type, choosing the NN learning algorith $\mathrm{m}$ and other parameters of NN. System user interface also contains elements for saving detected data signals, loading data form physical or optical storages, saving system output reports, etc.

The system allows:

- input signal visualization;

- detecting input signals informative parameters;

- performing input signal preprocessing;

- performing input data cluster analysis;

- determin ing and building separating hyperplanes;

- classifying the state of OUC and displaying the characteristics of its class;

- saving the values of selected informative parameters;

creating decision rule;

- converting obtained results to user-friendly format. 


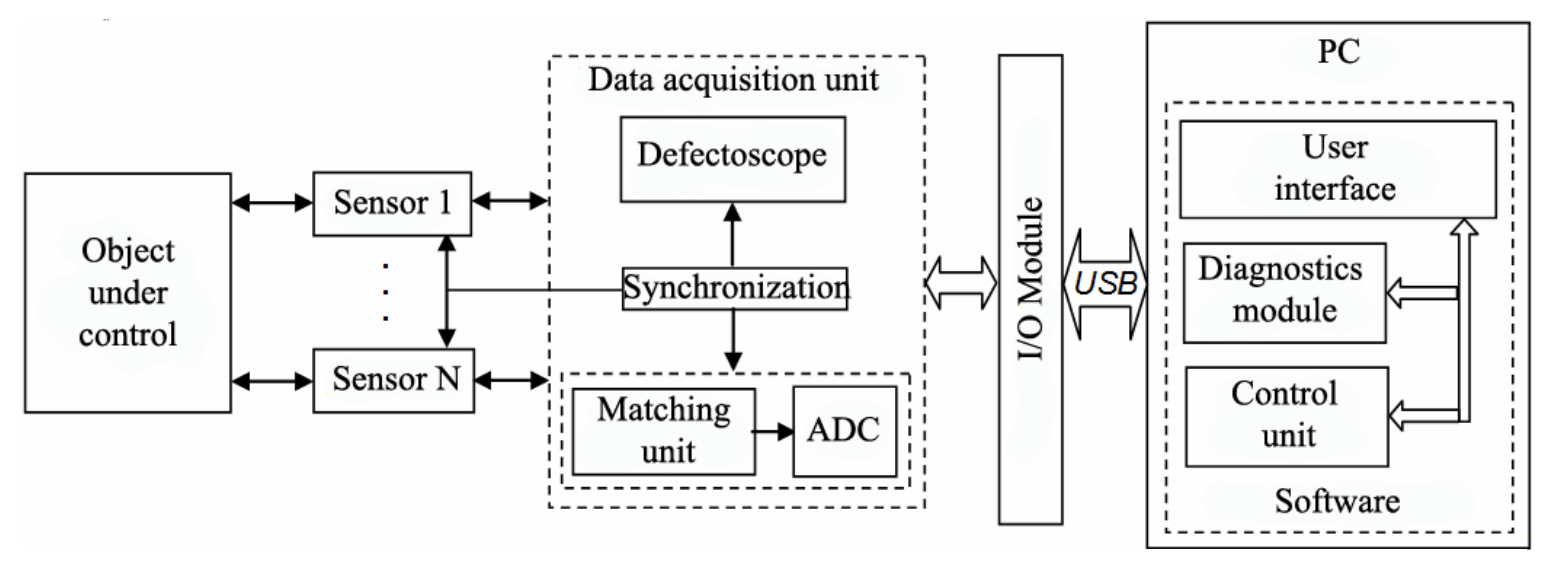

Figure 2. Standardless diagnostics system structure

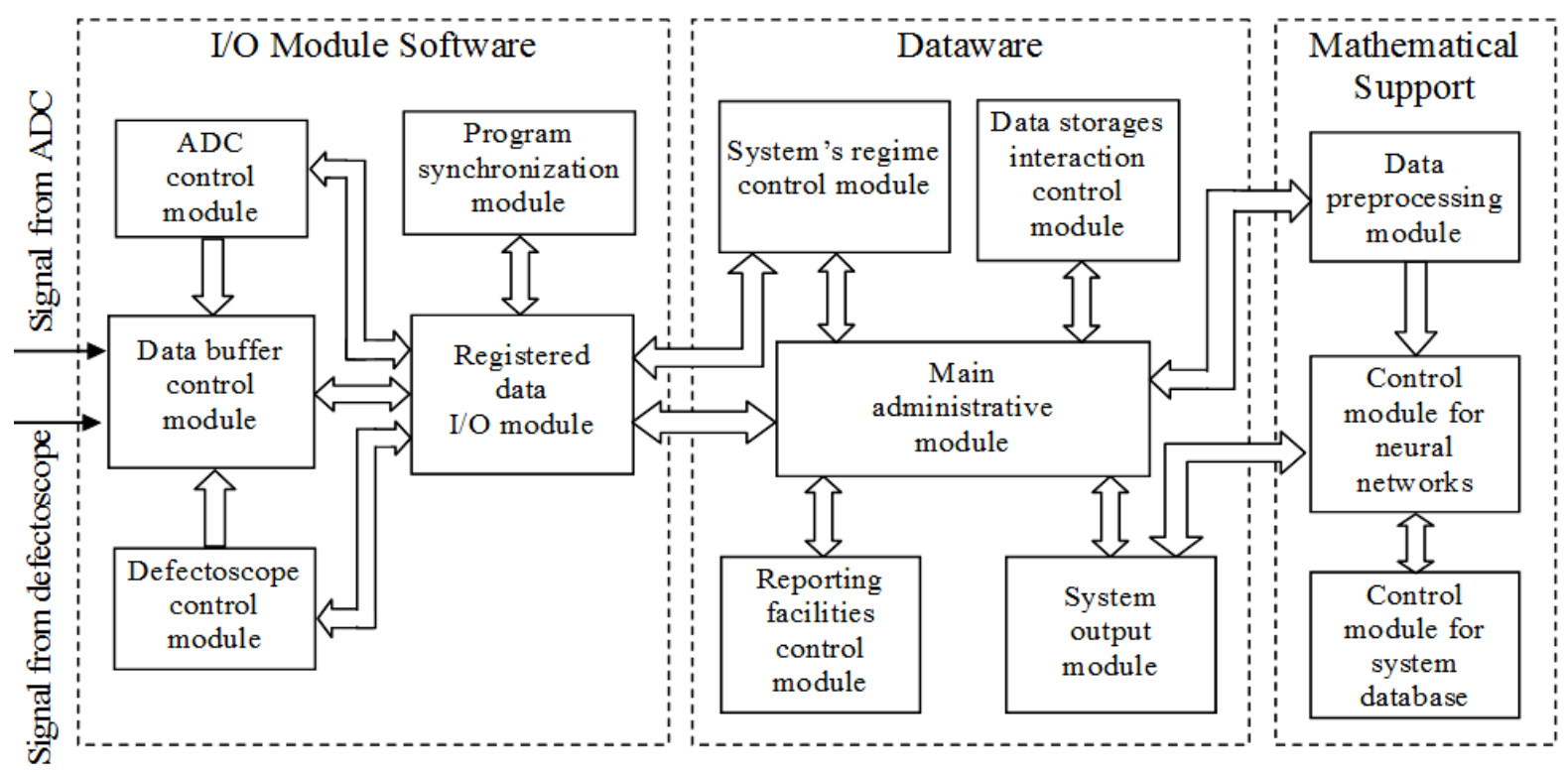

Figure 3. Nondestructive testing system software architecture

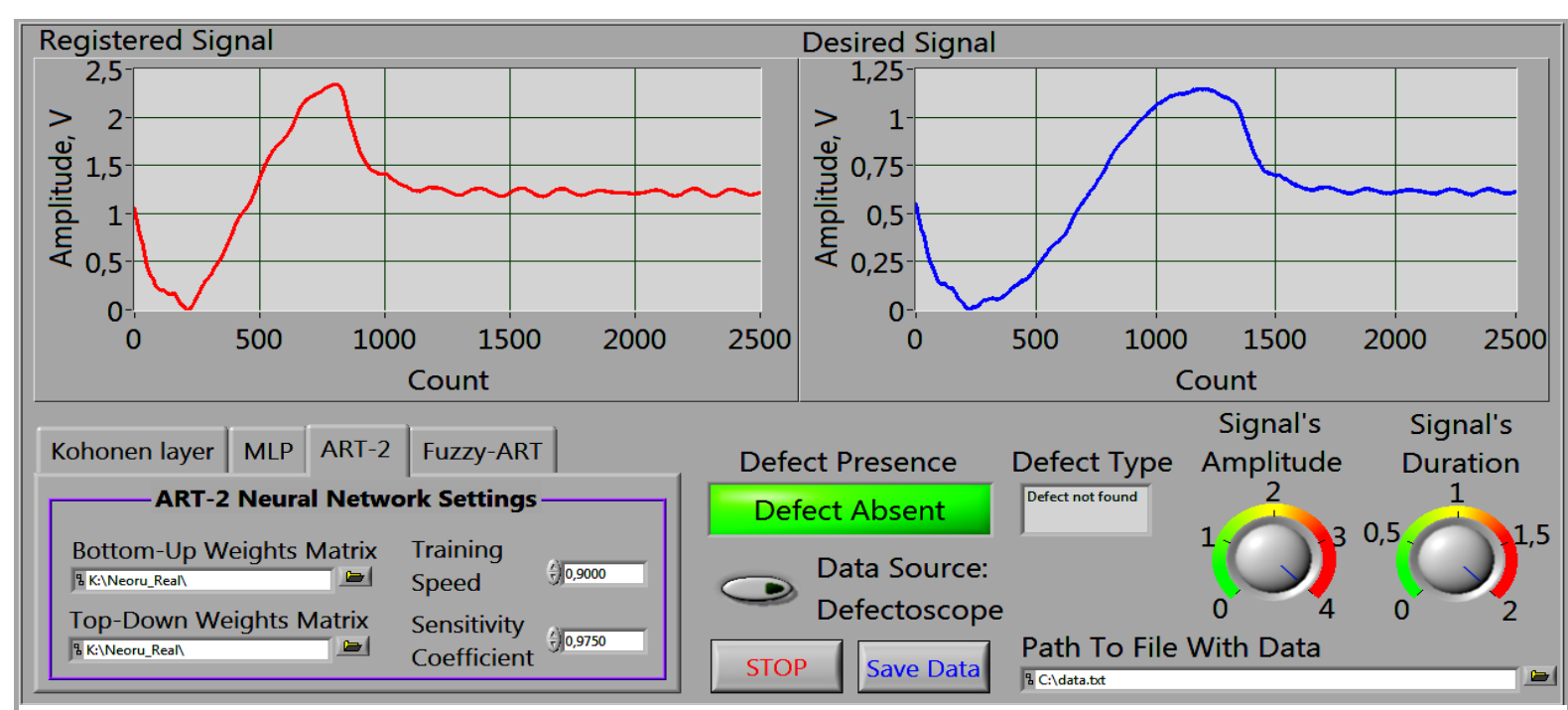

Figure 4. User interface of developed system 


\section{Neural Network Classifier}

Input data processing and signal classification is perform ed with usage of artificial neural networks. They are used due to fact that in the problems of nondestructive testing the NN allows to determine (classify) retrieved informative signals with high reliability and perform correct classification of new signals which were not previously presented to neural network during training. The usage of neural networks guaranties the high noise immunity, increases the reliability of control and entire system performance and decreases the system software and hardware costs.

The work of NN classifier which based on Kohonen NN (Kohonen layer) and multilayer perceptron could be described in such way. Firstly the multidimensional array which describe system input signal comes to classifier input from defectoscope, ADC or file stored on data storage. Next this array is processed by Kohonen layer (it forms classes and training samples for multilayer perceptron training). Formed by Kohonen layer classes and samples are used for setting up the weights of multilayer perceptron. Classificati on is performed by the multilayer perceptron. The special observing unit determines appearance of new or abnormal objects which do not belong to any of the existent in the NN me mo ry classes. In the case of such appearance the new class is formed in the system memory which corresponds for appeared object and the $\mathrm{NN}$ classifier performs reconfiguration. As a result there is no necessary to form the huge set of predefined etalon defects for system initial configuring. However the system reconfiguring during new class appearance requires additional time resources.

After retrieving from $\mathrm{NN}$ the information about defect class the system generates report about the result of nondestructive testing in the user-friendly format. It is stored on the data storage or displayed to operator.

The Kohonen layer is used due to ability to perform cluster analysis and forming initial training samples for multilayer perceptron training. The result of Kohonen layer work is a set of possible classes (defects of object under control). Next this set could be used for initial multilayer perceptron training. In contrast to the classical realization of Kohonen $\mathrm{NN}$ or Kohonen maps in which due to different class sizes every class is described by several neurons, in the developed system the other approach is used. Every class has its own center and orbit which determines the variance of the objects in this class and specifies the size of class. Thus the information about class (center and size) is contained only in one neuron from Kohonen layer. The Kohonen layer training is performed by WTA (winner takes all) method[37]. Each neuron in Kohonen layer determines the distance from new input object to class center, to class orbit and the quotient of these two values. Such algorithm allows define object class exactly. The lack of this approach is in concentrated NN memory, i.e. information about one class is stored in one neuron and in case of its crash information about entire class will be lost. This lack could be resolved by using additional copying of NN parameters. However this copying needs less me mo ry then approach with several neurons for one class but in cases of NN damage guaranties its reliability.

For defect classifying of objects from co mposite materials the multilayer perceptron with three layers and multiple numbers of neurons in each layer was implemented. The number of neurons in output layer depends on the number of possible object under control classes. The number of neurons in hidden layers depends on the complexity of separating hyperplanes for features spaces separation.

Every layer performs nonlinear transformation of the linear combination of input layer signal (output signal of previous layer) and its weights. Based on multilayer perceptron classifier allows solving the wide set of classification problems with different complexity and has high reliability of control and classification.

For multilayer perceptron training backpropagation algorith $\mathrm{m}$ [37] was used. Training set for this purposes was created by Kohonen layer in the train of the cluster analysis of reg istered data. Program listing of module which performs Kohonen layer training is shown in Fig. 6a. There are two main blocks in this module:

1) Distance finder block - evaluates value of the distance function. This value is used for calculating degree of similarity of the input vector and etalon vector in neural network's memory. Distance finder block implements the following distance functions: Euclidean, squared Euclidean distance, Manhattan distance, Chebyshev distance, Mahalanobis distance, Jeffries-Matusita distance etc. In more details this part of software is described in[24].

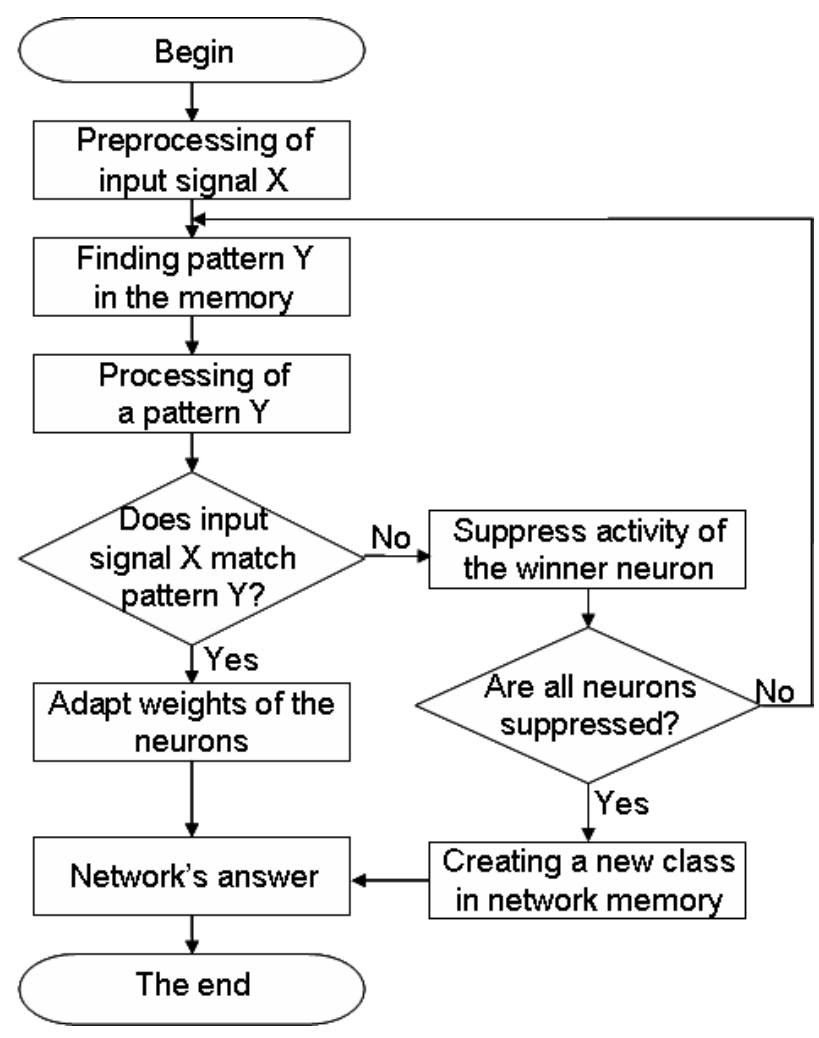

Figure 5. The ART neural net works algorithm 


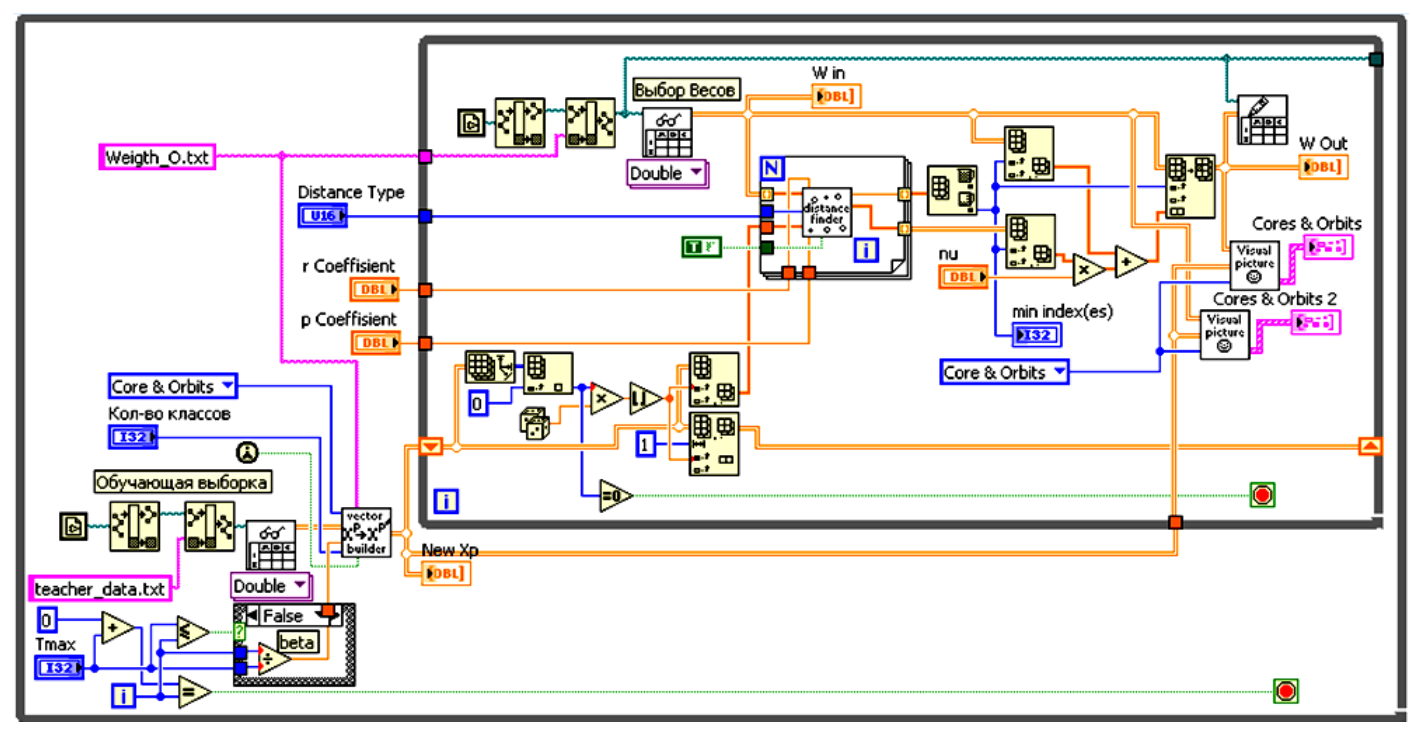

(a)

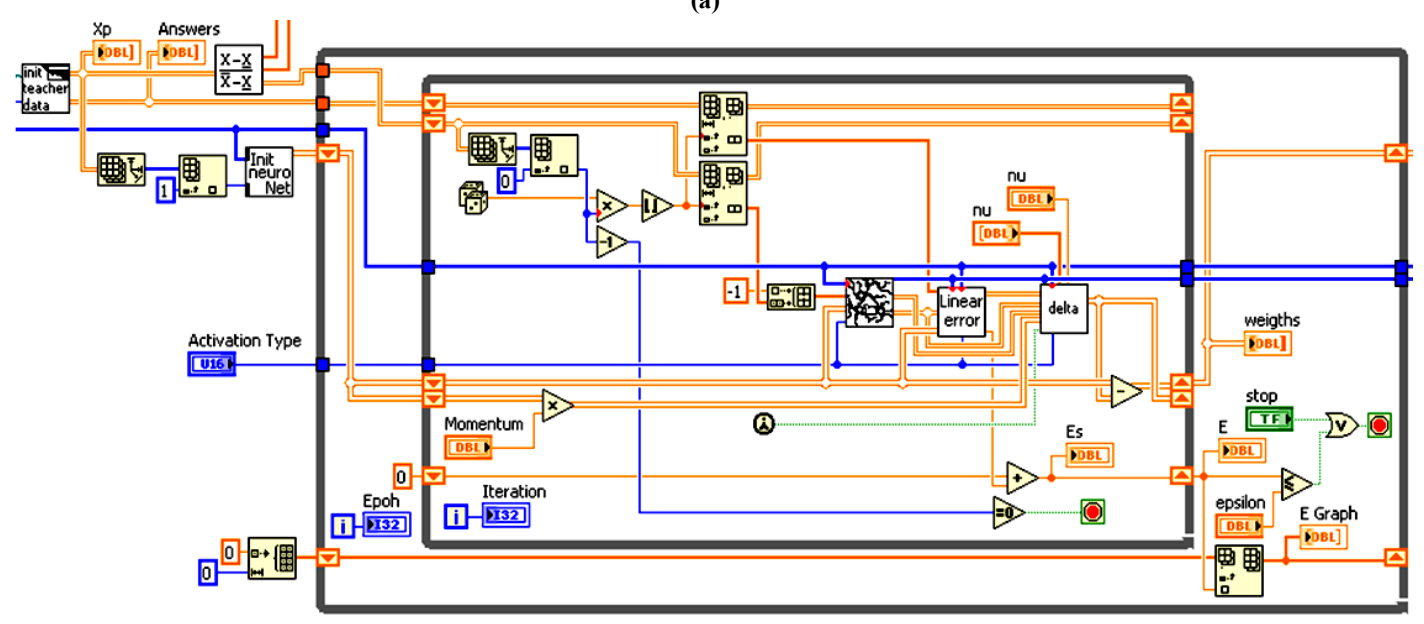

(b)

Figure 6. Program list ings of modules which perform Kohonen layer training (a) and mult ilayer perceptron training (b)

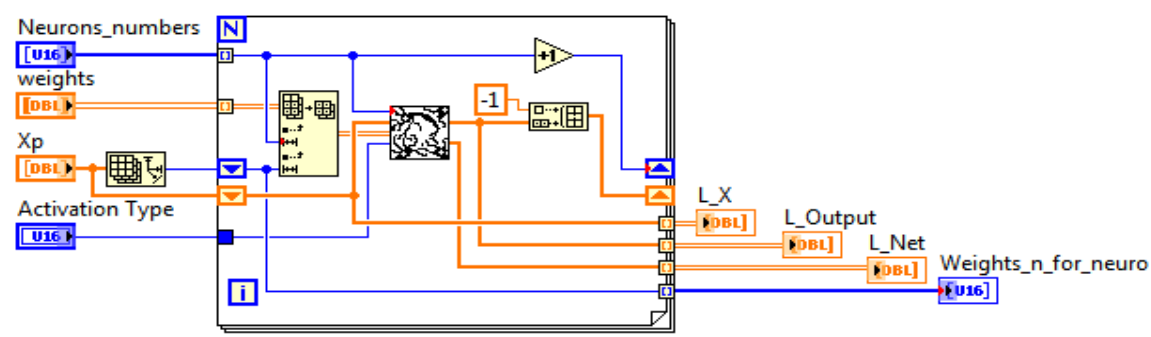

Figure 7. Program listing of module which implements multilayer perceptron

2) Transformation block - transforms input vector in way that is needed for implementation of the "convex combination" algorith $\mathrm{m}[38]$. Method of convex co mbination provides most optimal dis tribution of classes centers density.

For multilayer perceptron training it was developed program module which implemented some variety of gradient methods (Fig. 6b).
For neural network training it is possible to choose back propagation algorithm or one of its modifications (resilient propagation - RProp, second order algorith ms, training with moments etc.). Program module for multilayer perceptron training performs sequential iterative process of developed neural network training using one of training algorithms, allows visualizing an error function of multilayer perceptron 
training. Train ing process stops if value of error function is lower than some specified threshold value. Program listing of module which implements multilayer perceptron is presented in Fig. 7.

ART neural network[39] is a vector classifier which contains two layers of neurons (bottom-up layer or comparis on layer and top-down layer or recognition layer). The algorith m of the ART neural networks which are used in developed software for signals classification is shown on Fig. 5 .

During the classification process, the input signal is processing according to the weights of the neurons in comparis on layer of an ART-network. The signal is then fed to a recognition layer where the competitive search of the one single winning neuron (the neuron with an active output signal) is performed. Winner neuron is a neuron whose weight vector lies closest to the input vector (signal). This neuron, that becomes active under the influence of the input signal, corresponds to a particular class (pattern), which was formed in the memory of neural network and could include an input signal. On the next stage a feedback signal fro $m$ the winner neuron in the recognition layer comes back together with weights of an appropriate neuron to the comparison layer In the comparis on layer input signal is checked for compliance with the pattern in the network memory. If this pattern does not correspond to the input signal, the activity of the winner neuron in the recognition layer must be suppressed and the search phase for the new winner neuron (pattern) in the network memory should be performed again but without suppressed neuron. The algorithm is repeated until correct pattern is found or all neurons in the recognition layer are suppressed. If all neurons in the recognition layer are suppressed, then it means that the input signal belongs to the new pattern. This new pattern was not registered by neural network earlier and it didn't similar to any of the previously registered patterns, stored in the network me mo ry. In this case, the neural network will generate a new class and will provide a new neuron in the recognition layer. If during the search phase correct pattern (appropriate neuron with the corresponding weights) is found in the recognition layer, then the search phase stops and neural network generates as its output a signal that corresponds to one of the existing classes. Weights of the winning neuron in the recognition layer will be adapted according to appropriate network teaching rules[17,18,27-29]. So desired signal pattern in network memory will in more general form describe the group of signals which belong to the appropriate class.

For information signal processing the ART-2 and Fuzzy-ART neural networks were used. Classification of defects via the ART-2 and Fuzzy-ART neural networks is performed by analyzing changes of the registered signals shape, forming in the memory of neural network desired informative signal patterns which correspond to non-defect parts of controlled samples and comparing registered signals with generated patterns.

Program listing of modules which realized ART-2 and Fuzzy-ART neural networks is shown on Fig. 9.

\section{Experimental Part}

For the developed software testing were used samples of honeycomb panels which were provided by the state enterprise "Antonov" and were used in the manufacture of aircraft models "An":

Two samples (S1 and S2) of honeycomb panels from wings of aircraft type An-70 (Fig. 8a, 8b) of the material ELUR-P-0.1 and honeycomb filler PSP-1-2.5-45 with 10 $\mathrm{mm}$ of thickness with artificially formed defects. The following types of defects were under control: peeling skin on the inside of the filler cladding (Fig. 10a, 10b); control was made from the outside of the cladding;

One sample (S3) of the composite panel of aluminum alloy sheet gluing and sealing "viksint" which is used on the aircraft An-140 (Fig. 8c) with artificial defects like as delamination of the sealant. Control was made from the outside of the cladding (Fig. 10c).
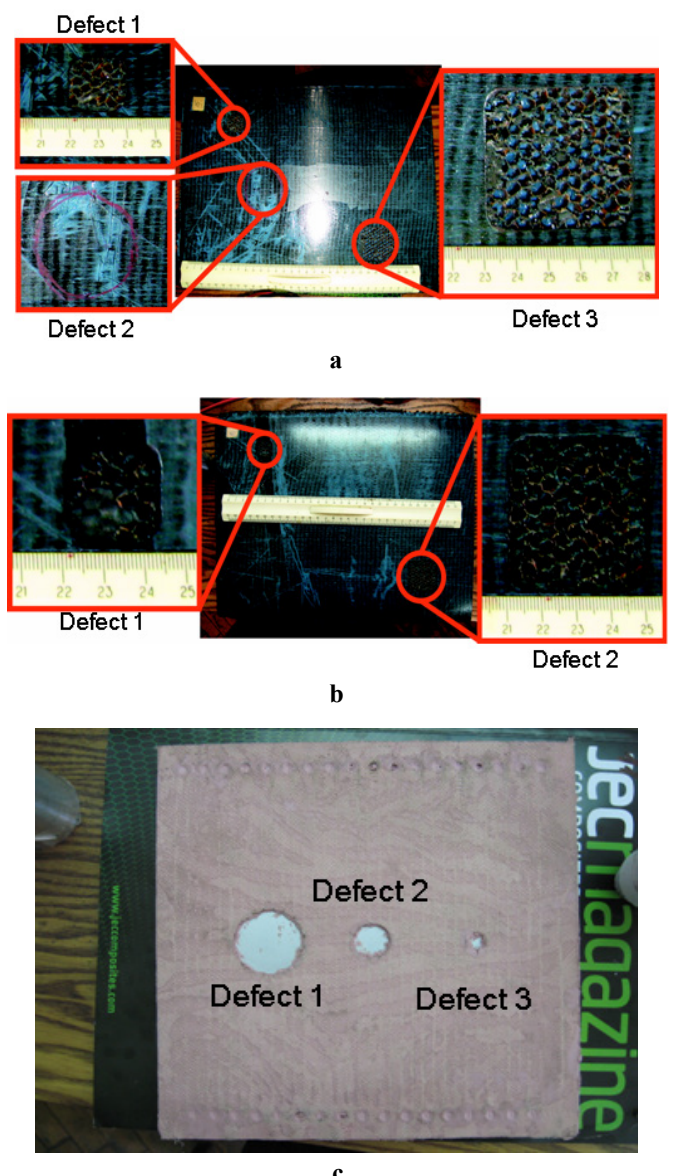

Figure 8. Samples $\mathrm{S} 1(a), \mathrm{S} 2(b)$ and $\mathrm{S} 3(c)$ of honeycomb panels (bottom view)

Areas of defects under control were divided on the points with distance between each point equal $2 \mathrm{~mm}$. Scanning was performed with a discrete step equal $2 \mathrm{~mm}$ in the direction indicated in Fig. 10. The numbers near the borders of areas with de fects mean number of scanning points, were area with defect is begun and ended. The developed software has been tested for the detection and diagnosis of defects in described samples of composite materials. 


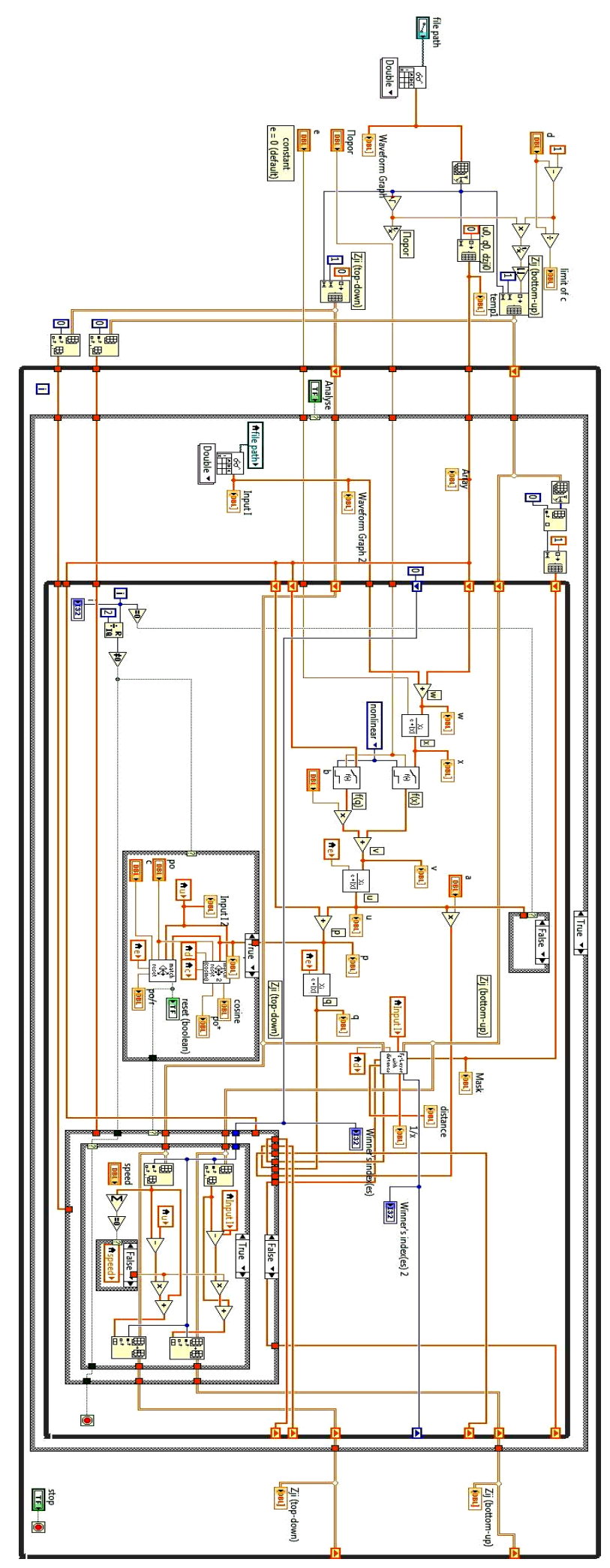

(a)

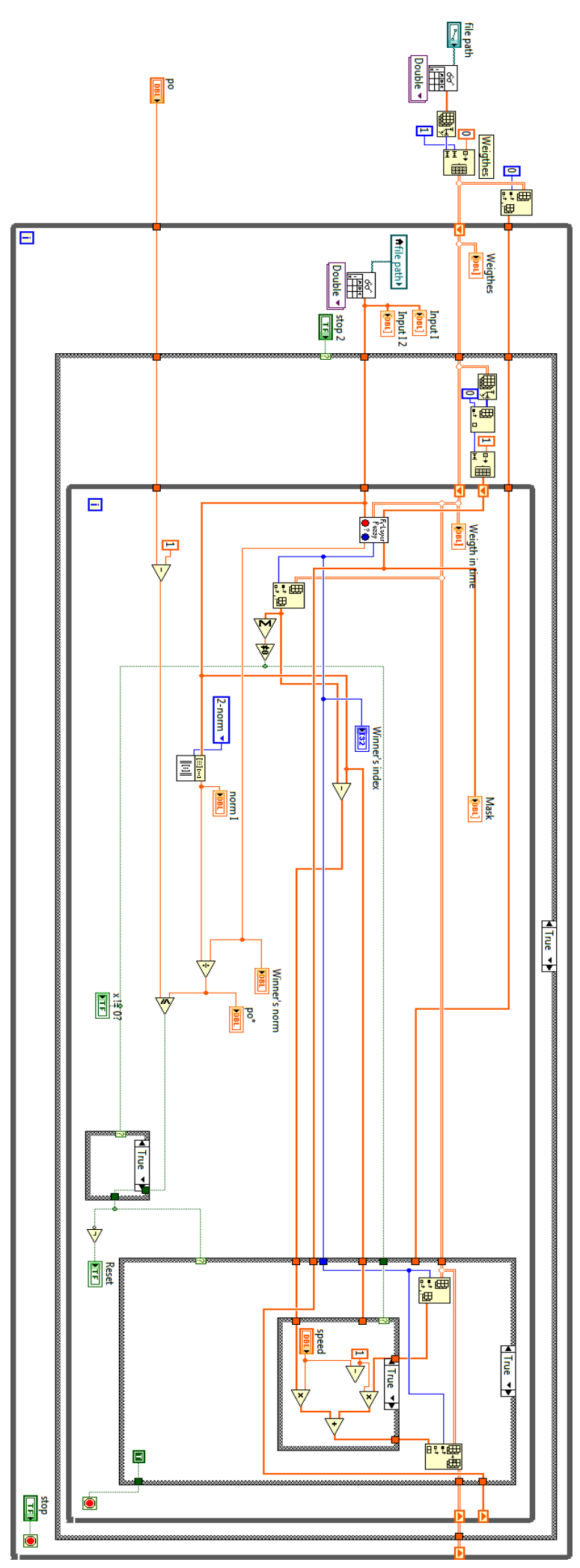

(b)

Figure 9. Program listing of modules which implements ART -2 (a) and Fuzzy-ART (b) neural net works

Thus, the diagnosis of defects in described samples of honeycomb panels with artificial defects and signals processing was performed $v$ ia three groups of methods:
1) Analyzing the amp litude and duration of the registered signals in individual and complex (by grouping these two parameters to the aggregate feature space) way, and 
constructing between different object states (defect - without defects) separating hyperplanes via a hybrid neural network $[4,5]$.

2) Statistical analysis of the registered signals, which includes spectral transformation of information signals and subs equent analys is of the spectral densities us ing chi-square distribution.

3) Analyzing the change of the registered signals shape via neural networks of adaptive resonance theory (ART-2 and Fuzzy-A RT) $[17,18]$, because changes of signals shape allow with high reliability detect defects presence in honeycomb panels and classify its type.

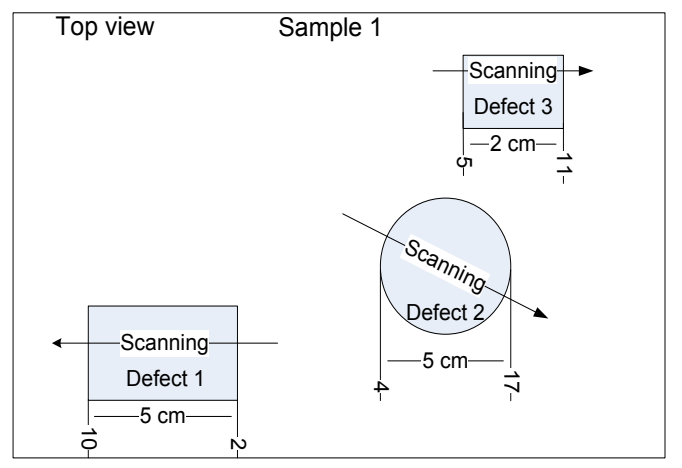

a
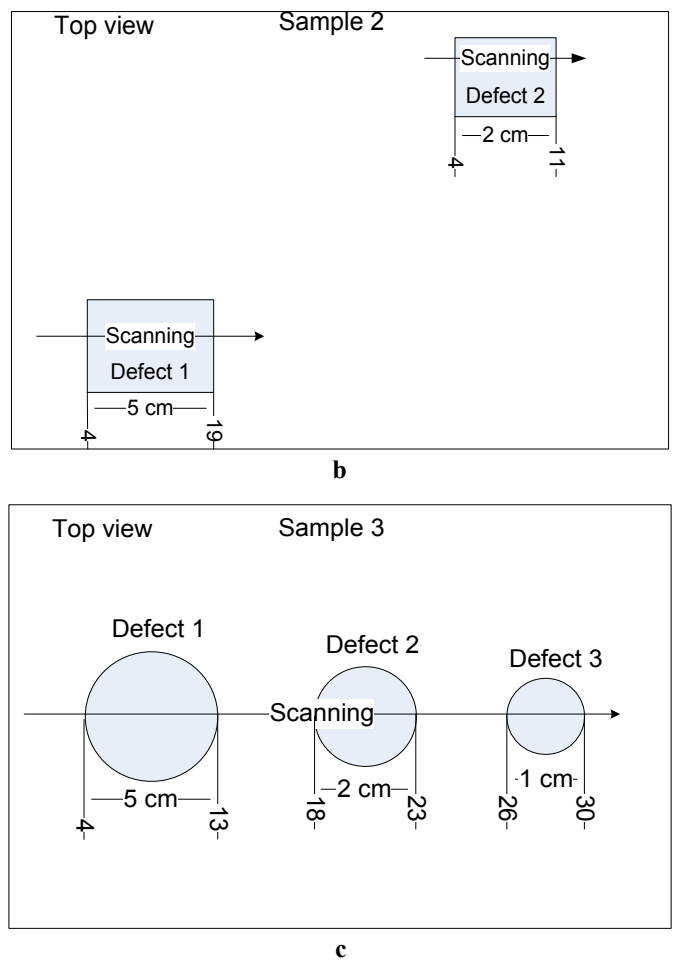

Figure 10. Location of defects in samples $\mathrm{S} 1(a), \mathrm{S} 2(b)$ and $\mathrm{S} 3(c)$. Scanning was made with step $2 \mathrm{~mm}$

For the nondestructive testing of described samples for each sample and each area (without defect and with different defects) 250 realizations of the informative signals were obtained (Fig. 11). During the control process registered signals were given one by one in a casual order to the neural network entrance. After an appropriate signal was given to system, it was removed from input samples collection. This procedure was repeated until the input samples collection became empty.
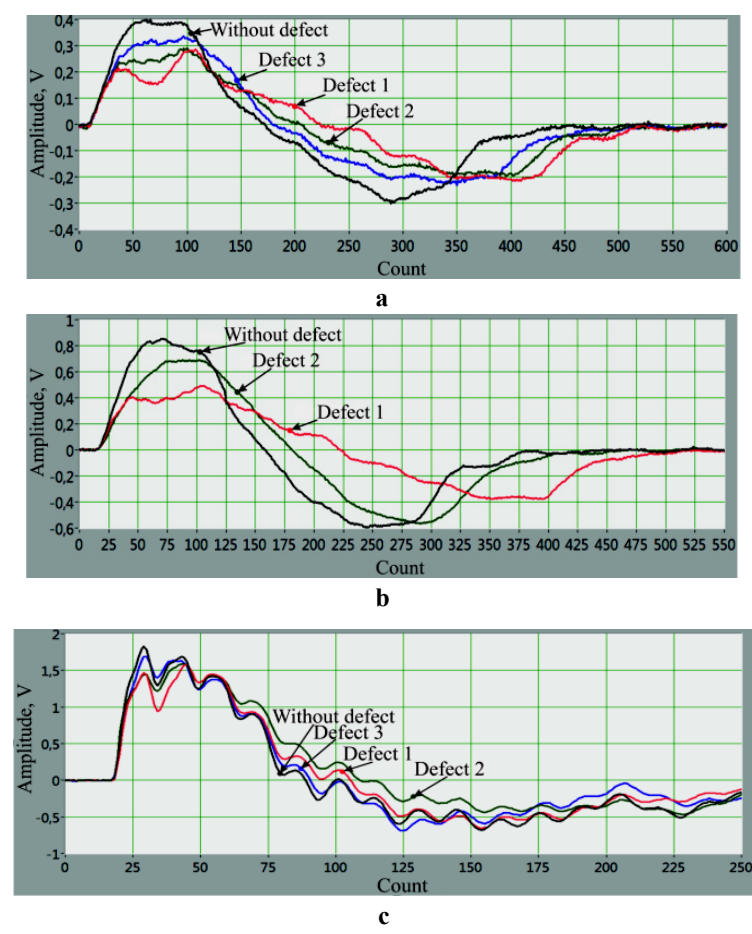

Figure 11. Informative signals which were obtained from areas without defects and areas with different defects during non-destructive test ing of honeycomb panel samples $\mathrm{S} 1(a), \mathrm{S} 2(b)$ and $\mathrm{S} 3(c)$
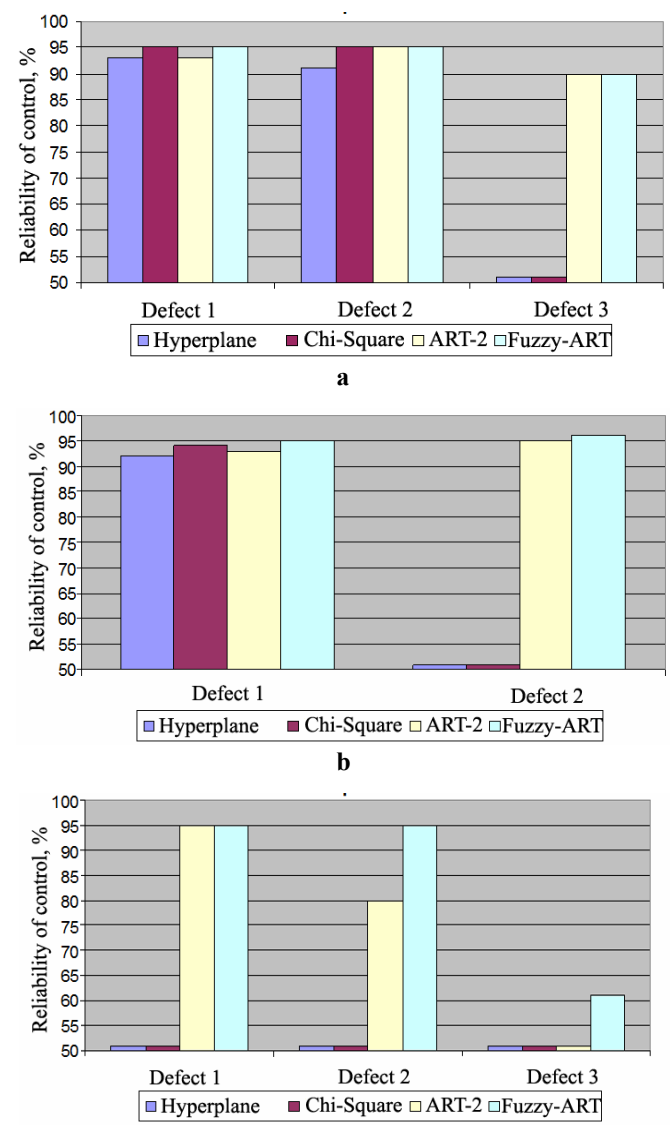

c

Figure 12. Reliability of control of honeycomb panel samples S1 (a), S2(b) and $\mathrm{S} 3(c)$ 
After the classifier has formed its data base the verification of the nondestructive testing reliability $v$ ia described software has been performed. For these purposes for each area 100 new signals were obtained and given to the neural network for classification. Obtained results of the nondestructive testing reliability of products fro $m$ composite materials using described signal processing methods are presented on Fig. 12.

Obtained results shows that the decision rule based on spectral transformation of information signals and subsequent statistical analys is of the spectral densities using chi-square distribution as well as the decision rule based on separating hyperplanes constructed in the coordinate system amp litude-duration via hybrid neural network failed to detect defects with plottage less then $2 \mathrm{~cm}^{2}$ (de fect 3 on the sample 1 and defect 2 on the sample 2). During control the sample 3 amp litude peaks are located on counts 7-12 and 20-23 that corresponds to areas with largest defects (defect 1 with plottage $5 \mathrm{~cm}^{2}$ and defect 2 with plottage $2 \mathrm{~cm}^{2}$ ). However, due to the low information content of signal's duration values, it is impossible to determine the presence of defects $1-3$ with plottage 5,2 and $1 \mathrm{~cm}^{2}$, respectively in the sample 3 using for signal processing groups of methods 1 and 2 . At the same time using the amplitude and duration of the data signals as informative parameters during controldefects with plottage greater then $5 \mathrm{~cm}^{2}$ (defect 1 on the sample 1 and sample 2) it was obtained reliability of the control about $90 \%$. Using spectral transformation and subsequent statistical analysis during control the same defects it was obtained reliability of the control about $90-93 \%$.

Usage of ART-2 and Fuzzy-A RT neural networks for the registered information signals processing during the control of honeycomb panels allows to determine bounds of defective areas 1 and 2 with plottage 5 and $2 \mathrm{~cm}^{2}$ respectively on the sample 1 and a defective area 1 with plottage $5 \mathrm{~cm}^{2}$ on the sample 2 . Also, unlike the groups of methods 1 and 2, classifier based on ART-2 and Fuzzy-ART neural networks has detected bounds of the defective areas with plottage $2 \mathrm{~cm}^{2}$ (defect 3 on the sample 1 and defect 2 on the sample 2). Also, with this classifier bounds of the two of three defective areas on the sample 3 were successfully detected (defect 1 and defect 2 with plottage 5 and $2 \mathrm{~cm}^{2}$ respectively). So, it could be extended that usage of clas sifier based on ART-2 and Fuzzy-ART neural networks in nondestructive testing of honeycomb panels allows to detect defects with plottage greater then $2 \mathrm{~cm}^{2}$ which is difficult or impossible with most currently used methods of diagnosis. The reliability of control via described classifier is over then $95 \%$.

\section{Conclusions}

Special software of nondestructive testing system was developed by authors. This software allows obtain more information about the technical state of complex objects under control, perform classification of possible defects and detect appearance of anomalies in material structure. Devel oped software increases the reliability of the object's technical state classification, reduces timetable and computationalburden for data signal processing and decision making.

For signal processing in developed software classifier based on hybrid neural network and neural network of adaptive resonance theory (ART-2 and Fuzzy-ART) is used. It allows provide non-linear separation and classification of objects by a set of diagnostic parameters, detect complex relationship between the damage degree of an object under control and informative parameters values. Described classifier during the training can automatically change its settings, reaching the highest reliability of the control, detect and classify subsurface defects in honeycomb panels with high reliability and accuracy, as well as defects that are located on the back side of the cladding with plottage larger than $2 \mathrm{~cm}^{2}$ and thickness of composite panel equal to 12.8 $\mathrm{mm}$. The reliability of the nondestructive testing via specified classifier is more than $95 \%$.

Developed software advantages are in its architecture flexibility, high performance and reliability of data signal processing, human-engineered interface. At the moment developed software is in pilot testing and used at the department of information-measuring systems of the National Aviation University for the experimental data processing obtained during the nondestructive testing of composite materials.

\section{REFERENCES}

[1] Cawley P. Clayton D.L. R. A vibration technique for the measurement of contact stiffness // Mechanical systems and signal processing. - № 1. - 1987. P. 273-283.

[2] Eremenko V.S. Information-measuring system for diagnostics of products from composite materials: $\mathrm{PhD}$ Thesis / V.S. Eremenko - Kiev, 2003. (In Ukrainian)

[3] Mokijchuk V.M. Diagnostics system of products from composite materials / V.M. Mokijchuk, V.S. Eremenko // Bulletin of the Khmelnytsky National University. - 2007. №2. - V.2. - P. 150-153. (In Ukrainian)

[4] Suslov E.F. The control system of products from composite materials / E.F. Suslov, V.S. Eremenko, V.M. Mokijchuk // «Educational, scientific and engineering applications in LabVIEW and National Instruments technology»: materials of the 8th international scientific conference, November 20-21, 2009.: proceedings. - Moscow, 2009. - P. 87-90. (In Russian)

[5] Benlarbi S. and Melo W. Polymorphism Measure for Early Risk Prediction. In Proceeding of 21st ICSE 1999, Los Angeles USA.

[6] Zeeshan, A. Towards Performance Measurement and Metrics based Analy sis of PLA Applications. Int. Jr. SE. \& App. 2010. $1(3): 66-80$.

[7] Zeeshan, A. Measurement Analysis and Fault Proneness Indication in Product Line Applications (PLA). Frontiers in 
Artificial Intelligence and Applications 2007, IOS Press, 161: 391-400. Scientific \& Academic Publishinghttp://www.sapu b.org

[8] Victor Basili, Jens Heidrich, Mikael Lindvall, Jürgen Münch, Myrna Regardie, Dieter Rombach, Carolyn Seaman, Adam Trendowicz. Linking Software Development and Business Strategy Through Measurement. IEEE Computer 2010, 43(4): $57-65$.

[9] Pereidenko A.V. Demerit rating system based on artificial neural networks / A.V. Pereidenko, Y.V.Kuts, V.S. Eremenko//'Days of nondestructive control 2010": matetials of XXV National Conference with International participation "Defektoskopiya'10", June 13-17, 2010.: proceedings Sophia,2010. - p.469-475. (In Russian)

[10] Pereidenko A.V. The system of standardless diagnosis of composite materials based on hybrid neural network./ A.V. Pereidenko, V.S. Eremenko, E.F. Suslov, P.A. Shegedin//", Educational, scientific and engineering applications in LabVIEW and National Instruments technology": materials of the 9th international scientific conference, December 3-4, 2010.: proceedings - Moscow,2010.-P. 207-212. (In Russian)

[11] Eremenko V.S. Application of linear recognition methods in problems of nondestructive testing of composite materials / V.S. Eremenko, O.O. Gileva// «Electromagnetic and acoustic methods of nondestructive testing of materials and products LEOTEST-2009»: materials of the 14th international scientific conference, February 16-21, 2009.: proceedings Slavskoe,2009.-P. 84-87. (In Ukrainian)

[12] Eremenko V.S. The study of defects in cellular screens low-frequency acoustic techniques / V.S. Eremenko, O.A. Gileva, V.Y.Derecha, E.F.Suslov, E.O.Pikolenko // Nauchni izvestia. - 2011. - №1. - P.49-51. (In Russian)

[13] Murashov V.V. Defects in parts of monolithic and multilayer structures made of polymer composite materials and methods for their detection / V.V. Murashov, A.F. Rumyancev // Control. Diagnostics. - 2007. - №4,5. - P. 23-32. (In Russian)

[14] Eremenko V.S. Standardless inspection of composite materials / V.S. Eremenko, V.M. Mokijchuk // Physical methods and means to control the environment, materials and products. Nondestructive testing of materials and structures.2009. - №14. - P. 63-70. (In Ukrainian)

[15] Eremenko V.S. Methods for constructing decision rules for nondestructive testing of composite materials / V.S. Eremenko, V.Y.Derecha, O.A. Gileva, E.D. Bliznuk // «Nondestructive Testing and Technical Diagnostics»: materials of the 6th international scientific conference, June 9-12, 2009.: proceedings. - Kiev, 2009. - P. 359-363. (In Russian)

[16] Eremenko V.S. Aspects of construction of decision rules in the nonstandard inspection of composite materials / V.S. Eremenko, O.A. Gileva, J.O. Pavlenko // “ Instrument 2008: status and prospects": »: materials of the 6th international scientific conference, April 22-23, 2008.: proceedings. - Kiev, 2008. - P. 227-228. (In Russian)

[17] Pereidenko A.V. Uses of the modified ART-2 neural network architecture in the nondestructive testing system of products from composite materials / A.V. Pereidenko, V.S. Eremenko, O.V. Monchenko // "Modern methods and instruments of nondestructive testing and technical diagnosis": materials of 19th International Conference, October 3-7, 2011.: proceedings - Gurzuf, 2011. - p. 81-84. (In Ukrainian)

[18] Pereidenko A.V. System Of Standartless Diagnostic Of Cell Panels Based On Fuzzy-ART Neural Network / A.V. Pereidenko, V.S. Eremenko, V.O. Rogankov // " MRRS-2011": Proceedings of the Third Microwaves, Radar and Remote Sensing Symposium, August 25-27, 2011: proceedings. - Kyiv, 2011. - p. 181-183.

[19] Barhatov V.A. Detection of defects using artificial neural network of a special type / V.A. Barhatov // Defectoscopy.2006. - №2. - P. 28-39. (In Russian)

[20] Naidenko A.G. Determining the reliability of the diamond cutters composite superhard materials by recording and analyzing acoustic emission: PhD Thesis / A.G. Naidenko. Kiev, 2009. (In Ukrainian)

[21] Zajickiy O.V. Recognition of aircraft en gin es blades state via neural networks during vibroacoustic monitoring: $\mathrm{PhD}$ Thesis / O.V. Zajickiy. - Kiev, 2008. (In Ukrainian)

[22] Barhatov V.A. Detection of signals and their classification using pattern recognition / V.A. Barhatov // Defectoscopy. 2006. - №4. - P. 14-27. (In Russian)

[23] Pereidenko A.V. Construction of decision rules in multiparameter NDT / A.V. Pereidenko, V.S. Eremenko, O.A. Gileva, E.F.Suslov // «Modern methods of of nondestructive testing and technical diagnosis»: materials of the 18th international scientific conference, October 5-9, 2010.: proceedings. - Yalta, 2010. - P. 78-81. (In Ukrainian).

[24] Pereidenko A.V. Investigation of cluster analysis algorithms for solving nondestructive testing tasks/ A.V. Pereidenko, V.S. Eremenko// East European journal of advanced technologies. -2010. -№1/5(43). - P. 40-43. (In Ukrainian)

[25] Pereidenko A.V. The system of cluster analysis of nondestructive testing products from composite materials/ A.V. Pereidenko, V.S. Eremenko, V.A Rogankov// Knowledge-intensive technologies. -2010. -№3. - P. 73-77. (In Ukrainian)

[26] Pereidenko A.V. The system of defects classification based on artificial neural networks / A.V. Pereidenko, V.S. Eremenko, J.O. Pavlenko // Bulletin of the National Technical University of Ukraine "Kyiv Polytechnic Institute". Series 'Instrument Engineering'. - 2010. - №40. - p. 72-80. (In Ukrainian)

[27] Carpenter G.A. ART 2: Stable self-organization of pattem recognition codes for analog input patterns / G.A. Carpenter, S. Grossberg // Applied Optics. - 1987. - №26. - P. 4919-4930.

[28] Carpenter G.A. ART 2-A: An adaptive resonance algorithm for rapid category leaming and recognition / G.A. Carpenter, S. Grossberg, D.B. Rosen // Neural Networks. - 1991. - №4. - P. 493-504.

[29] Carpenter G.A. Fuzzy ART: Fast stable learning and categorization of analog patterns by an adaptive resonance system / G.A. Carpenter, S. Grossberg, D.B. Rosen // Neural Networks. - 1991. - №4. - P. 759-771.

[30] Sumathi S., Surekha P. Computational Intelligence Paradigms: Theory \& Applications using MATLAB. - CRC Press, Inc., 2010. $-851 \mathrm{p}$.

[31] http://www.neurosolutions.com/ 
[32] http://neuropro.ru/

[33] Borovikov V.P. STATISTICA Neural Networks: The methodology and technology of modern data analysis. Moscow: Hotline Telecom, 2008. - 392p. (In Russian)

[34] Fudoseev V.P. Digital Signal Processing in LabVIEW. Moscow: DMK Press, 2007. - 472p. (In Russian)

[35] Zagidulin R.Sh. LabVIEW in research and development. Moscow: Hotline Telecom, 2005. - 352p. (In Russian)

[36] Eremenko V.S. Detection of impact damage honeycomb panels using low-velocity impact/ V.S. Eremenko, V.M.
Mokijchuk, A.M. Ovsankin// Technical diagnostics and nondestructive testing. - 2007. -№1. - P. 24-27. (In Russian)

[37] Haykin Simon Neural Networks - A Comprehensive Foundation, Second Edition. - Pearson Education, Inc., 2005. $-823 \mathrm{p}$.

[38] Zaincev I.V. Neural Networks: main models. - Voronej, 1999. -76 p. (In Russian)

[39] Carpenter G.A. A massively parallel architecture for a self-organizing neural pattern recognition machine / G.A. Carpenter, S. Grossberg // Computer Vision, Graphics, and Image Processing. - 1987. - №37. - P. 54-115. 